\title{
When should we intervene to control the 2009 influenza $\mathrm{A}(\mathrm{H} 1 \mathrm{~N} 1)$ pandemic?
}

H Sato (hsato@ndmc.ac.jp) ${ }^{1,2}$, H Nakada ${ }^{3,2}$, R Yamaguchi, ${ }^{4,2}$, S Imoto ${ }^{4,2}$, S Miyano ${ }^{4}$, M Kami3 $^{3}$

1. Department of Medical Informatics, National Defense Medical College Hospital, Saitama, Japan

2. These authors contributed equally to this work

3. Division of Social Communication System for Advanced Clinical Research, Institute of Medical Science, University of Tokyo, Tokyo, Japan

4. Human Genome Center, Institute of Medical Science, University of Tokyo, Tokyo, Japan

Citation style for this article:

Citation style for this article: Sato H, Nakada H, Yamaguchi R, Imoto S, Miyano S, Kami M. When should we intervene to control the 2009 influenza A(H1N1) pandemic?. Euro Surveill. 2010;15(1):pii=19455. Available online: http://www.eurosurveillance.org/ViewArticle.aspx?Articleld=19455

We simulated the early phase of the 2009 influenza $A\left(H_{1} N_{1}\right)$ pandemic and assessed the effectiveness of public health interventions in Japan. We show that the detection rate of border quarantine was low and the timing of the intervention was the most important factor involved in the control of the pandemic, with the maximum reduction in daily cases obtained after interventions started on day 6 or 11. Early interventions were not always effective.

\section{Background}

In Japan, the 2009 influenza $A\left(\mathrm{H}_{1} \mathrm{~N}_{1}\right)$ pandemic raised questions as to whether the Japanese government's response was adequate. In the early phase, Japan concentrated on onboard quarantine inspection at international airports rather than other public health interventions. From 28 April 2009, the Ministry of Health, Labour and Welfare restricted passenger entry and conducted onboard quarantine inspections to delay the import of influenza cases for as long as possible. At Narita International Airport, the largest international airport in Japan, onboard quarantine inspection was carried out until 18 June 2009, and more than two million passengers from Canada, the United States and Mexico were screened. Ten cases were confirmed by RT-PCR and 60 contacts were quarantined. Other countries also conducted border quarantine inspections. Taiwan, for example, screened 1,328,645 passengers from 29 April to 4 June, and four cases were confirmed [1].

While large amounts of material and human resources were invested in this preventative measure, the first patient infected with the pandemic influenza $A\left(\mathrm{H}_{1} \mathrm{~N}_{1}\right)$ virus in Japan, detected on 16 May, had no overseas travel history. This observation indicated that infected individuals had passed the onboard quarantine inspection undetected or had already entered Japan before the inspection was initiated. While some experts suggested that onboard quarantine inspection was not effective, others argued that it delayed the import of pandemic influenza cases and increased the time to prepare for a response. The validity of the Japanese government's early response to the novel type of influenza virus remains controversial.

In this study, we estimated the number of imported cases of pandemic influenza that passed the border quarantine undetected. The domestic pandemic caused by these cases was simulated using mathematical simulation modelling to assess the optimal public health intervention to the influenza pandemic in the early pandemic phase in Japan.

\section{Methods}

We simulated indigenous transmission of pandemic influenza, caused by cases undetected by the onboard quarantine inspection, in a community of 100,000 individuals. The daily number of undetected cases was estimated by the daily number of detected cases among passengers entering Japan, the distribution of incubation periods, and that of infectious periods [2]. To simulate domestic transmission, we modified the SEIR (susceptible, exposed, infected, recovered) model [3] to take into account undetected cases as exogenous input (SEIRix model). Intervention is also taken into account in the model. Details of the SEIRix model can be found in a document provided on the following website: http://bonsai.ims.u-tokyo.ac.jp/ imoto/ suppl_contN1H1.html. We set both incubation time and infectious period to 3.5 days [4-6]. The reproduction number was set as 2.3 obtained from a study conducted in Japan [7].

The simulation settings were as follows: At the border quarantine, some of the symptomatic cases were detected and isolated; undetected cases entered into the country and transmitted the virus to susceptible individuals. The first day on which a case of pandemic influenza was detected in Japan was defined as day 0 . To compare the effects of the timing of public health interventions, we examined four different initiation dates, namely, day 1 , day 6 , day 11 , and day 16 after the first case of the virus was detected at the border 
quarantine. An intervention was defined as an action aimed at reducing the chances of susceptible individuals having contact with infectious cases, which included school closures or governmental orders to the population to stay at home. The scale of the intervention was described by the compliance rate of staying at home, which was set at three levels in the simulation, i.e. small (10\%), medium (30\%), and large (50\%). Susceptible individuals who stayed at home were assumed to have no contact with infectious individuals. Individuals who stayed at home did so for three, seven or 14 days from the start of the intervention.

The maximum number of symptomatic cases per day and the time of their detection were used to evaluate the effectiveness of the intervention, because the acute capacity of medical institutions would be related to these endpoints, rather than to the total number of cases diagnosed during the pandemic. The total number of individuals who received an intervention was multiplied by the duration of the intervention and the product was called the person-day. The person-day of each intervention was divided by that of the smallest intervention, which was started on day 1 and lasted for three days. We defined this ratio as the standardised person-day ratio. The standardised person-day ratio was used as a surrogate marker for the resources needed for a given intervention. The relationship between effectiveness and required resources was also assessed for each intervention.

\section{Results}

In our simulation, border quarantine inspection detected the first case of $\mathrm{H}_{1} \mathrm{~N}_{1}$ influenza in Japan 56

\section{TABLE 1}

Estimated number of undetected cases of pandemic influenza A(H1N1) among flight passengers entering Japan

\begin{tabular}{|c|c|c|c|c|c|}
\hline & $\boldsymbol{\psi}$ & $\boldsymbol{r}$ & $\boldsymbol{\eta = 1 . 2}$ & $\boldsymbol{\eta = 1 . 2 4}$ & $\boldsymbol{\eta}=\mathbf{1 . 3}$ \\
\hline \multirow{3}{*}{ RL-IP } & 1 & 0.0000984 & 16.838 & 17.094 & 17.465 \\
\cline { 2 - 6 } & 0.7 & 0.0001405 & 27.483 & 27.848 & 28.379 \\
\hline \multirow{2}{*}{$2-I P$} & 1 & 0.0001801 & 31.381 & 32.169 & 33.327 \\
\cline { 2 - 6 } & 0.7 & 0.0002572 & 48.259 & 49.384 & 71.038 \\
\hline \multirow{2}{*}{$3.5-I P$} & 1 & 0.0005703 & 67.135 & 70.913 & 76.763 \\
\cline { 2 - 6 } & 0.7 & 0.0008147 & 99.336 & 104.732 & 113.09 \\
\hline
\end{tabular}

The table shows the number of infected passengers who could not be detected by the entry screening for representative values of $\eta$, where $\eta$ is the growth rate of the numbers of infected individuals on each day, $\psi$ is the detection rate of passengers who make in-flight progression and $r$ is the proportion of the infected passengers estimated by the number of detected infected passengers. We tested three variations of the incubation period: RL-IP has 1.4 days median period, that is the same incubation period as that in Rvachev and Longini (1985) and was used in Pitman et al. (2005) [2,8], 2-IP is an incubation period with a median period equal to two days and 3.5 -IP has 3.5 days median period. Detailed information on the methods used for the estimation can be found at http://bonsai.ims.u-tokyo.ac.jp/ imoto/suppl_contN1H1.html.

\section{TABLE 2}

Peak reduction and lag by intervention, simulation of pandemic interventions, Japan

\begin{tabular}{|c|c|c|c|c|c|c|c|c|c|c|c|c|}
\hline \multirow{4}{*}{ Scale } & \multicolumn{12}{|c|}{ Start date } \\
\hline & \multirow{2}{*}{\multicolumn{3}{|c|}{$\begin{array}{c}\text { Day } 1 \\
\text { Duration (days) }\end{array}$}} & \multirow{2}{*}{\multicolumn{3}{|c|}{$\begin{array}{c}\text { Day } 6 \\
\text { Duration (days) }\end{array}$}} & \multirow{2}{*}{\multicolumn{3}{|c|}{$\begin{array}{c}\text { Day } 11 \\
\text { Duration (days) }\end{array}$}} & \multirow{2}{*}{\multicolumn{3}{|c|}{$\begin{array}{c}\text { Day } 16 \\
\text { Duration (days) }\end{array}$}} \\
\hline & & & & & & & & & & & & \\
\hline & 3 & 7 & 14 & 3 & 7 & 14 & 3 & 7 & 14 & 3 & 7 & 14 \\
\hline \multicolumn{13}{|l|}{ Small $(v=0.1)$} \\
\hline Peak reduction ${ }^{a}$ & 0.99 & 0.97 & 0.90 & 0.98 & 0.94 & 0.84 & 0.97 & 0.91 & 0.88 & 0.96 & 0.96 & 0.96 \\
\hline Peak lag (days) ${ }^{\mathrm{b}}$ & 1 & 2 & 3 & 1 & 2 & 3 & 1 & 1 & -1 & 0 & -1 & -1 \\
\hline Standardised person-day ratio & 1.0 & 2.3 & 4.7 & 0.9 & 2.1 & 4.2 & 0.8 & 1.8 & 3.6 & 0.6 & 1.4 & 2.8 \\
\hline \multicolumn{13}{|l|}{ Medium $(v=0.3)$} \\
\hline Peak reduction $^{\mathrm{a}}$ & 0.97 & 0.91 & 0.78 & 0.95 & 0.85 & 0.64 & 0.91 & 0.76 & 0.73 & 0.92 & 0.92 & 0.92 \\
\hline Peak lag (days) ${ }^{b}$ & 2 & 4 & 9 & 2 & 4 & 9 & 2 & 4 & -5 & -3 & -3 & -3 \\
\hline Standardised person-day ratio & 3.0 & 7.0 & 14.0 & 2.7 & 6.4 & 12.7 & 2.3 & 5.4 & 10.8 & 1.8 & 4.2 & 8.3 \\
\hline \multicolumn{13}{|l|}{ Large $(v=0.5)$} \\
\hline Peak reduction $^{a}$ & 0.95 & 0.87 & 0.73 & 0.91 & 0.77 & 0.56 & 0.85 & 0.69 & 0.69 & 0.91 & 0.91 & 0.91 \\
\hline Peak lag (days) ${ }^{\mathrm{b}}$ & 3 & 7 & 15 & 3 & 8 & 17 & 3 & -8 & -8 & -4 & -4 & -4 \\
\hline Standardised person-day ratio & 5.0 & 11.7 & 23.3 & 4.5 & 10.6 & 21.2 & 3.8 & 9.0 & 18.0 & 3.0 & 6.9 & 13.9 \\
\hline
\end{tabular}

v: the compliance rate of staying at home.

a Peak reduction: the reduction rate of the maximum number of cases per a day compared with that of no intervention.

b Peak lag: the lag of the date when the maximum cases were observed compared with that of no intervention; negative values indicate that the peak was achieved earlier than in a scenario with no intervention. 
days after the report of the first case worldwide in Mexico. Our estimation suggests that at the time the first case was detected in Japan, more than 100 cases had already entered the country. The detection rate ranged from $7.1 \%$ to $22.3 \%$ (Table 1 ).

Small interventions were only minimally effective in reducing the maximum number of daily symptomatic cases and delaying the epidemic peak, regardless of the start date and duration of the intervention. When $10 \%$ of the susceptible individuals stayed at home for 14 days from day 1 , the maximum number of daily symptomatic cases was reduced by only $10 \%$ and the epidemic peak was delayed by three days (Table 2). A large-scale intervention for 14 days starting on day 6 was the most effective. This intervention reduced the maximum number of symptomatic cases by $44 \%$ and delayed the epidemic peak by 17 days (Table 2). Comparing any combination of intervention scale and duration indicated that the maximum reduction in daily cases was obtained from those interventions that started on day 6 or day 11. Medium interventions for three days starting on day 11 were more effective than those that were started on day 6 . Intriguingly, the earliest start date, day 1 , did not give the best outcome within the same duration or scale.

Large and long interventions with different starting dates showed differing pandemic curves (Figure). When $50 \%$ of susceptible individuals received an early intervention to stay at home for 14 days, a second increase in the pandemic was observed from the end of the intervention. When the intervention was started on day 1 or day 6 , the maximum daily number of symptomatic cases was obtained at the second peak, not

\section{FIGURE}

Simulation of pandemic curves after intervention to the susceptible population, Japan

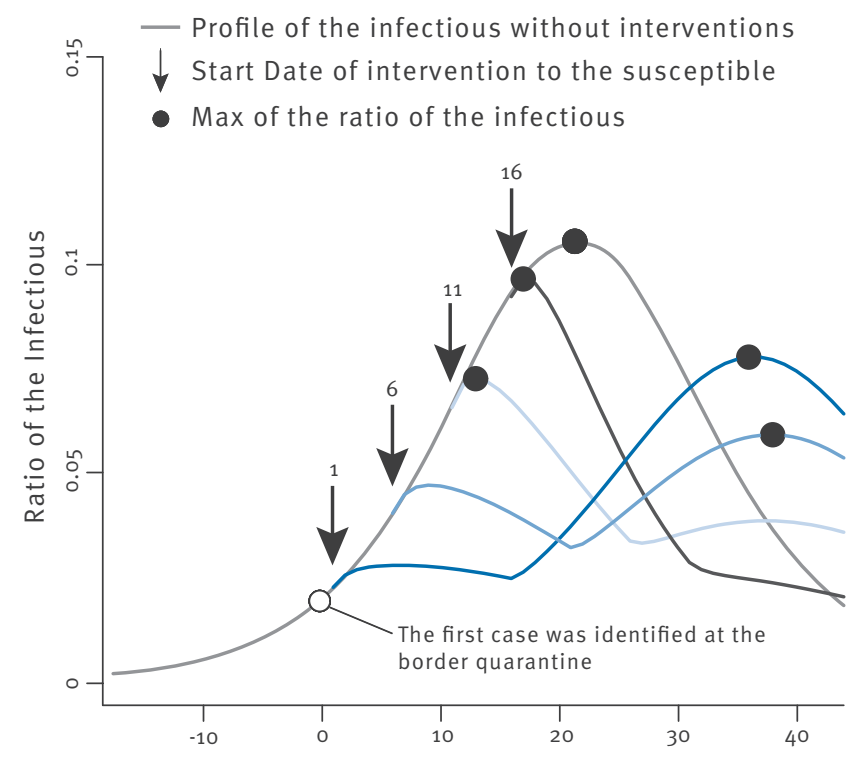

Day the first peak that was observed just after the intervention. The last intervention, started on day 16 , did not show a second peak, and the curve of the infection rate was attenuated.

The standardised person-day ratio showed that the most effective intervention, which reduced a peak to 56 percent, required the second largest amount of resources (Table 2). The most expensive intervention was a large intervention for 14 days starting from day 1. However, a medium intervention for 14 days starting from day 11 resulted in the same reduction in influenza cases with only half the amount of resources.

\section{Discussion}

We simulated the early phase of the 2009 influenza $A\left(\mathrm{H}_{1} \mathrm{~N}_{1}\right)$ pandemic in Japan and assessed the effectiveness of public health interventions. Our estimation of cases undetected in onboard quarantine inspections demonstrated the low detection rate of this technique. A previous study suggested that border quarantine inspection could not prevent importation of the virus completely [2]. Tomba and Wallinga also showed the low detection rate of border quarantine by mathematical modelling [9]. On 28 April 2009, the World Health Organization (WHO) advised that no restriction of regular travel or closure of borders be implemented against the pandemic influenza virus. Our results are consistent with these views. To effectively slow the epidemic curve, the Japanese public health responses to the pandemic influenza virus would have had to shift the emphasis from onboard quarantine inspection to active surveillance and preparation, and such interventions would have been necessary as soon as the first case of the virus was detected by the onboard quarantine inspection. However, the simulation of viral transmission showed that early initiation of an intervention is not always effective in reducing the maximum number of daily cases, as a secondary increase in influenza cases was observed after the implementation of the early intervention. Even if the public health intervention was large and long, the start date was crucial in maximising its effectiveness.

An adequately large and long intervention cannot always be implemented, because of the limitations of human and material resources. Furthermore, interventions against emerging infectious diseases may cause social and economic harm, even if the pandemic does not increase in severity [10]. Therefore, the public health agency and the government must plan a response policy based on scientific data, considering effectiveness, feasibility, and impact on economic or social activities. We used the standardised person-day ratio as an indicator of required resources and showed various patterns of effectiveness versus resources. For example, the most effective intervention required 21.2 times the resources of the smallest intervention. Using such an indicator, the government would have to assess the optimal policy in terms of their ability to implement it and its effect on the spread of the 
infection. In Japan, for example, decisions on school closure are taken independently by each school, but scientific forecasts would be able to support a decision for community-wide school closures while taking into consideration the effect on the influenza pandemic and its impact on society and economics. Cauchemez et al. reviewed the multiple aspects of school closure as a public health policy [11].

Most interventions to control pandemics were based on pessimistic scenarios [10]. Indeed, the onboard quarantine inspection in Japan was based on the response policy against the highly pathogenic avian influenza $A\left(\mathrm{H}_{5} \mathrm{~N}_{1}\right)$ and attempted to block all cases of the pandemic influenza $A\left(\mathrm{H}_{1} \mathrm{~N}_{1}\right)$ virus from entering the country. However, this method was ineffective in preventing the spread of the infection, and scientific policy-making would have been needed to minimise the adverse effects of this intervention $[12,13]$. In the current study, we have highlighted a method of accomplishing evidence-based public health policy making for emerging infectious diseases.

\section{Acknowledgements}

We thank Dr. Moriyo Kimura and Dr. Norihiko Yamada for their helpful comments and suggestions.

\section{References}

1. Influenza $A\left(\mathrm{H}_{1} \mathrm{~N}_{1}\right)$ central epidemics command center, Taiwan. Update on novel influenza $A\left(\mathrm{H}_{1} \mathrm{~N}_{1}\right)$ infection in humans. Taiwan: June 6 2009. Available from: http://flu.cdc.gov.tw/ public/Data/966124502.pdf

2. Pitman RJ, Cooper BS, Trotter CL, Gay NJ, Edmunds WJ. Entry screening for severe acute respiratory syndrome (SARS) or influenza: policy evaluation. BMJ. 2005;331(7527):1242-3.

3. Kermack WO, McKendrick AG. Contributions to the mathematical theory of epidemics. Proceedings of the Royal Society Series A. 1927;115:700-21.

4. Dawood FS, Jain S, Finelli L, Shaw MW, Lindstrom S, Garten $\mathrm{RJ}$, et al. Emergence of a novel swine-origin influenza $A\left(\mathrm{H}_{1} \mathrm{~N}_{1}\right)$ virus in humans. N Engl J Med. 2009;360(25):2605-15.

5. Nishiura $\mathrm{H}$, Inaba $\mathrm{H}$. Prediction of infectious disease outbreak with particular emphasis on the statistical Issues using transmission model,. Proceedings of the Institute of Statistical Mathematics. 2006;54(2):461-80.

6. World Health Organization. Considerations for assessing the severity of an influenza pandemic. Wkly Epidemiol Rec. 2009 May 29;84(22):197-202.

7. Nishiura H, Castillo-Chavez C, Safan M, Chowell G. Transmission potential of the new influenza $A\left(\mathrm{H}_{1} \mathrm{~N}_{1}\right)$ virus and its age-specificity in Japan. Euro Surveill. 2009;14(22):pii=19227. Available from: http://www. eurosurveillance.org/ViewArticle.aspx?Articleld=19227

8. Rvachev L, Longini I. A mathematical model for the global spread of influenza. Mathematical Biosciences. 1985;75(1):3-22.

9. Scalia Tomba G, Wallinga I. A simple explanation for the low impact of border control as a countermeasure to the spread of an infectious disease. Math Biosci. 2008;214(1-2):70-2.

10. Doshi P. Calibrated response to emerging infections. BMJ. 2009;339:b3471. doi: 10.1136/bmj.b3471.

11. Cauchemez S, Ferguson NM, Wachtel C, Tegnell A, Saour $G$, Duncan B, et al. Closure of schools during an influenza pandemic. Lancet Infect Dis. 2009;9(8):473-81.

12. Fineberg HV, Wilson ME. Epidemic science in real time. Science. 2009;324(5930):987.

13. Lipsitch M, Riley S, Cauchemez S, Ghani AC, Ferguson NM. Managing and reducing uncertainty in an emerging influenza pandemic. N Engl J Med. 2009;361(2):112-5. 